\title{
Temporal Evaluation of Cardiac Myocyte Hypertrophy and Hyperplasia in Male Rats Secondary to Chronic Volume Overload
}

\author{
Yan Du, Eric Plante, Joseph S. Janicki, \\ and Gregory L. Brower \\ From the Department of Cell Biology and Anatomy, School of \\ Medicine, University of South Carolina, Columbia, South Carolina
}

The temporal myocardial remodeling induced by chronic ventricular volume overload in male rats was examined. Specifically, left ventricular (LV) cardiomyocyte length and width, sarcomere length, and number of nuclei were measured in male rats $(n=8$ to 17$)$ at $1,3,5,7,21,35$, and 56 days after creation of an infrarenal aortocaval fistula. In contrast to previously published reports of progressive increases in cardiomyocyte length and cross-sectional area at 5 days post-fistula and beyond in female hearts, cardiomyocyte length and width did not increase significantly in males during the first 35 days of volume overload. Furthermore, a significant decrease in cardiomyocyte length relative to age-matched controls, together with a reduced number of sarcomeres per cell, was noted in male hearts at $\mathbf{5}$ days post-fistula. There was a concurrent increase in the percentage of mononucleated cardiomyocytes from $11.6 \%$ to $18 \%$ at 5 days post-fistula. These initial differences could not be attributed to cardiomyocyte proliferation, and treatment with a microtubule stabilizing agent prevented them from occurring. The subsequent significant increase in $\mathrm{LV}$ weight without corresponding increases in cardiomyocyte dimensions is indicative of hyperplasia. Thus, these findings indicate hyperplasia resulting from cytokinesis of cardiomyocytes is a key mechanism, independent of hypertrophy, that contributes to the significant increase in LV mass in male hearts subjected to chronic volume overload. (Am J Pathol 2010, 177:1155-1163; DOI: 10.2353/ajpath.2010.090587)

The myocardium is remodeled in response to a sustained increase in stress and/or injury in an effort to normalize myocardial wall stress. ${ }^{1}$ This compensatory process is typically progressive and involves all components of the myocardium. Depending on the nature of the stress, cardiomyocyte remodeling involves the parallel and/or in- series addition of sarcomeres, resulting in myocyte thickening and/or lengthening, respectively. ${ }^{2}$ In response to ventricular volume overload induced secondary to an aortocaval fistula, Liu et $\mathrm{al}^{3,4}$ reported progressive, temporally proportional increases in cardiomyocyte length and cross-sectional area in female rats. However, our recent studies have found gender differences in the pattern of global myocardial remodeling, in which males develop eccentric hypertrophy and heart failure in response to a sustained ventricular volume overload, whereas the female heart is cardioprotected, developing concentric hypertrophy and remaining compensated. ${ }^{5-9}$

These observations raise the obvious question-does this difference in the pattern of remodeling exist at the level of the cardiomyocyte as well? Thus, the purpose of this study was to determine whether temporal differences in cardiomyocyte size and structure contribute to adverse remodeling occurring in male hearts subjected to a sustained volume overload and compare those findings with that previously reported for female rats. ${ }^{3,4}$ To this end, we examined isolated cardiomyocytes from adult, male rats at discrete times over an 8-week period of chronic volume overload. The results indicate that the myocardial remodeling process induced by chronic volume overload in male hearts is largely due to cardiomyocyte hyperplasia resulting from cytokinesis of differentiated adult cardiomyocytes.

\section{Materials and Methods}

All experiments were performed using cardiac tissue from adult, male Sprague-Dawley (Hsd:SD) rats. Groups consisting of rats that had undergone surgery to create an infrarenal aortocaval fistula as previously described, ${ }^{5}$ and age-matched sham-operated controls $(n=9)$ were sacrificed at $1(n=17), 3(n=8), 5(n=13), 7(n=11)$, $21(n=14) 35(n=9)$ and 56 days $(n=16)$. Tissue

Supported in part by NIH National Heart, Lung and Blood Institute grants HL073990 and HL62228 (J.S.J.).

Accepted for publication May 27, 2010.

Address reprint requests to Gregory L. Brower, D.V.M., Ph.D., Cell Biology and Anatomy, School of Medicine, University of South Carolina, Columbia, SC 29208, E-mail: Gregory.Brower@uscmed.sc.edu. 
samples were collected and assigned random identification numbers for subsequent analysis to blind the observers as to the source of the sample. All studies conformed to the principles of the National Institutes of Health Guide for the Care and Use of Laboratory Animals and the protocol was approved by our Institution's Animal Care and Use Committee.

\section{Potassium Hydroxide Cardiomyocyte Isolation}

Cardiomyocytes were isolated using a modified potassium hydroxide $\mathrm{KOH}$ procedure initially described by Gerdes et al $^{10}$ Briefly, formalin fixed tissue from a midlevel transmural section of the left ventricle (LV) was minced into $1 \mathrm{~mm}^{3}$ pieces, placed into a $0.1 \mathrm{~mol} / \mathrm{L} \mathrm{KOH}$ solution, and allowed to stand undisturbed at room temperature for 24 hours. The tissue was then placed in a 0.1 $\mathrm{mol} / \mathrm{L}$ PBS solution and continuously agitated for $10 \mathrm{~min}-$ utes. Cardiac myocytes were purified using a 10\% Ficoll density gradient. Finally, the cells were resuspended in $10 \%$ buffered formalin and cytospun for 5 minutes at 10,000 rpm onto a microscope slide that was processed with gallocyanin $(0.15 \%)$ and chromium potassium sulfate $(5 \%)$ staining to better visualize cellular architecture. ${ }^{11}$

\section{Enzymatic Cardiomyocyte Isolation}

Adult Sprague-Dawley rats were anesthetized and the descending thoracic aorta cannulated proximal to the first set of intercostal arteries. The heart was removed and attached to a modified Langendorff perfusion apparatus via the cannula. The heart was initially perfused with a $37^{\circ} \mathrm{C}$ calcium-free modified Joklik's MEM solution (10.0 mmol/L HEPES Sodium Salt, $25.0 \mathrm{mmol} / \mathrm{L} \mathrm{NaHCO} 3,1.2 \mathrm{mmol} / \mathrm{L}$ $\mathrm{MgSO}_{4}$ ) for 5 minutes and then perfused with a $37^{\circ} \mathrm{C}$ Joklik's solution with Type II collagenase (Worthington Biochemical Corp., Lakewood, NJ) until adequate digestion had occurred. Next, the heart was removed from the perfusion apparatus and the LV minced and mechanically triturated in a collagenase solution. The dissociated cells were fixed for 10 minutes in $2 \%$ paraformaldehyde and cytospun onto slides for immunofluorescence.

\section{5-Bromo-2'-Deoxyuridine Labeling and Paclitaxel Treatment}

Groups of rats ( $n=5$ per group) received bromodeoxyuridine (BrdU; Sigma Chemical Company, St Louis, MO), $50 \mathrm{mg} \cdot \mathrm{kg}^{-1}$ body weight via daily intraperitoneal (i.p.) injection from 0 to 5 days, from 0 to 7 days, or from 5 to 7 days post-fistula, to investigate the possibility of myocyte hyperplasia. At the end of these study periods, the hearts were removed and cardiac myocytes were isolated by enzymatic digestion as described above. Cardiomyocytes were fixed for 10 minutes in $2 \%$ paraformaldehyde and then cytospun onto slides for immunofluorescence. Incubation with the primary monoclonal antiBrdU antibody (Roche Applied Science; Penzberg,
Germany) was performed over a 1 hour period at room temperature; F-actin was stained with Alexa488 conjugated phalloidin (Invitrogen Co. Carlsbad, CA); and nuclei were stained with 4',6-diamidino-2-phenylindole (Sigma Chemical Co., St. Louis, MO).

The chemotherapeutic agent paclitaxel (Sigma) stabilizes microtubules and as a result interferes with cell division. Paclitaxel was administered at a dosage of 20 $\mathrm{mg} \mathrm{kg}^{-1}$ body weight in an attempt to prevent the occurrence of hyperplasia. ${ }^{12}$ Groups of rats received paclitaxel as daily i.p. injections from 0 to 5 days $(n=5)$ and from 5 to 8 days $(n=5)$ post-fistula. The hearts were then fixed in formalin and cardiac myocytes isolated using the $\mathrm{KOH}$ digestion technique described above. Microtubular structure was labeled using rabbit polyclonal anti- $\alpha$-tubulin (Santa Cruz Biotechnology, Inc; Santa Cruz, CA). After washing in PBS, the sections were incubated with a Cy3conjugated goat anti-rabbit IgG secondary antibody (Jackson ImmunoResearch Laboratories, Inc., West Grove, PA). Cardiomyocytes were also labeled for F-actin and with 4',6diamidino-2-phenylindole as described above.

\section{Cardiomyocyte Morphometry}

Light microscopic examination $(\times 200)$ was used to analyze 50 randomly chosen, intact rod-shaped cardiac myocytes. Images of the stained isolated myocytes were digitized and measurements consisting of unloaded, unstressed cell length, cell width, sarcomeric length, and sarcomere number were obtained using ImagePro Plus analysis software (Media Cybernetics, Inc., Bethesda, $\mathrm{MD})$. There was little difficulty in distinguishing true monoor binucleated cardiomyocyte cells. Damaged cardiomyocytes identified based on broken or fragmented cell membranes that were no longer intact were omitted from the analysis. Use of this exclusion criterion produced highly reproducible results with insignificant variation between observers. In addition, to validate the cellular dimensions obtained from the $\mathrm{KOH}$ isolated cardiomyocytes, cardiomyocyte cross section dimensions were measured in $5 \mu \mathrm{m}$ LV cross-sections stained with H\&E in multiple regions judged to be cut perpendicular to the long axis of the cells by the nearly round shape of capillaries in the region. Cardiomyocyte width was determined by digitizing myocyte cross-sectional areas, and long and short axis cell width were averaged to obtain mean cardiomyocyte diameter in 50 cells. All morphometric measurements were performed in a blinded manner.

\section{Scanning Electron Microscopy}

Sample preparation for scanning electron microscopy was performed according to the method described by Murphy. ${ }^{13}$ LV tissue samples were fixed for 2 hours in $3 \%$ glutaraldehyde in $0.1 \mathrm{~mol} / \mathrm{L}$ sodium cacodylate buffer $(\mathrm{pH} 7.4)$, and then $2 \%$ aqueous $\mathrm{OsO}_{4}$ for 2 hours. After rinsing, samples were treated for 12 hours with $8 \%$ glutaraldehyde $/ 2 \%$ tannic acid at $4^{\circ} \mathrm{C}$, followed by rinsing and a 2-hour treatment with $2 \% \mathrm{OsO}_{4}$. The glutaraldehyde/tannic acid and $\mathrm{OsO}_{4}$ treatment sequence was re- 
A

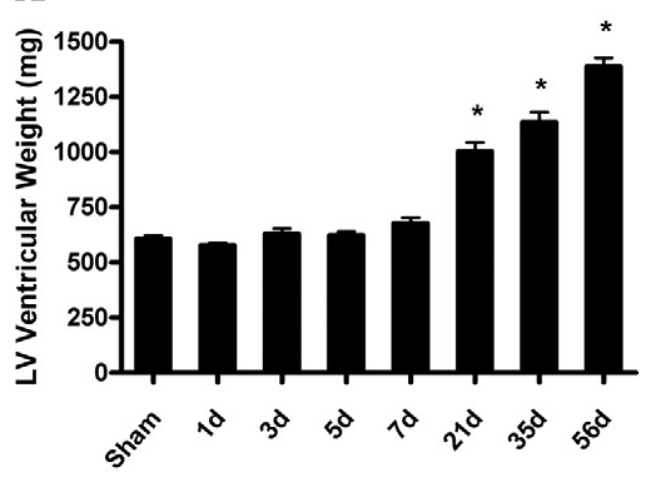

B

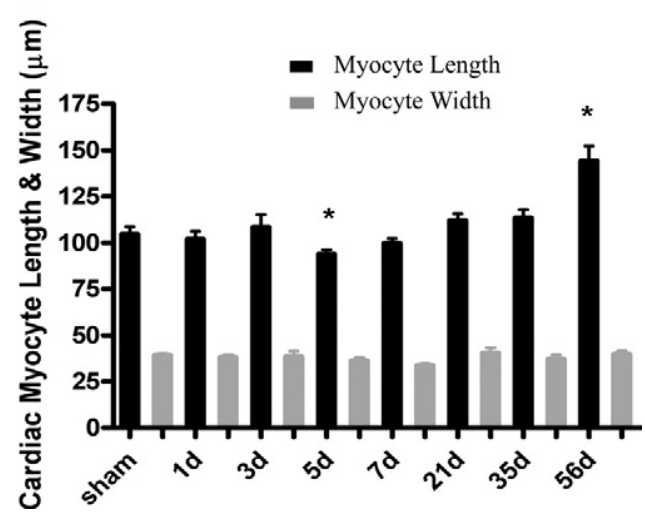

Figure 1. This graph depicts changes in left ventricular (LV) mass (A) and average cardiomyocyte length and width (B) over the temporal course following the creation of an aortocaval fistula. Data are mean \pm SEM. ${ }^{*} P \leq$ 0.01 vs. age-matched sham-operated control.

peated one more time. Samples were then dehydrated, dried, mounted, and viewed on a JEOL JSM-6300V (Tokyo, Japan) at $10 \mathrm{kV}$.

\section{Statistical Analysis}

All results are presented as means \pm SEM. Statistical analyses were performed using SPSS 11.5 Software (SPSS, Inc; Chicago IL). LV weight, cardiomyocyte length and width, and sarcomeric length were compared among the groups using analysis of variance. Intergroup comparisons were analyzed using a Bonferroni post hoc test. Statistical significance was taken to be $P \leq 0.05$.

\section{Results}

\section{Cardiac Mass}

LV weights for the various groups are shown in Figure $1 \mathrm{~A}$. Although LV weight remained relatively constant during the first week post-fistula, the subsequent development of myocardial hypertrophy was progressive, becoming significantly different from the sham operated control group at 21 days post-fistula and beyond $(P \leq 0.01)$.

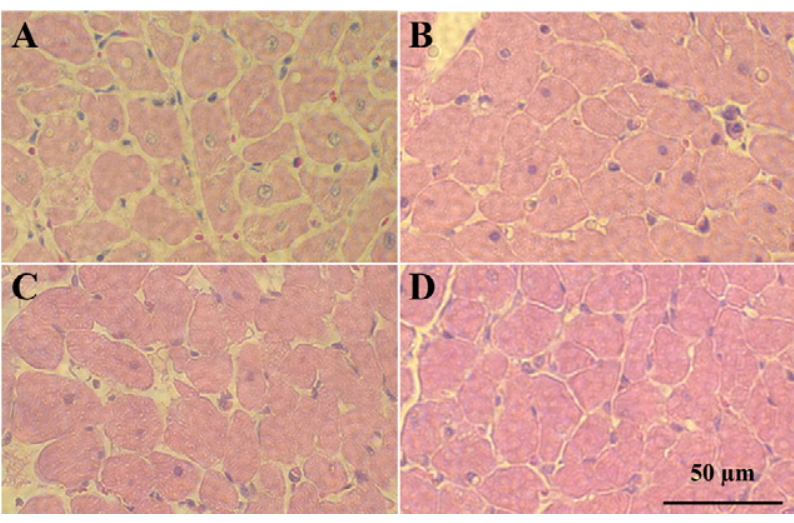

Figure 2. Representative photomicrographs depicting similar distribution of cardiomyocyte diameter temporally following the creation of an aortocaval fistula. Mean cardiomyocyte diameter post-fistula was not significantly different from the age matched sham-operated control group $(21.3 \pm 2.4$ vs. $22.3 \pm 1.5 \mu \mathrm{m}$ and $24.0 \pm 1.8$ vs. $25.0 \pm 1.7 \mu \mathrm{m}$ for the control and fistula groups at five days $(\mathbf{A}$ and $\mathbf{B})$ and 56 days $(\mathbf{C}$ and $\mathbf{D})$, respectively. Scale bar $=50 \mu \mathrm{m}$.

\section{Cardiomyocyte Remodeling}

Average cardiomyocyte length and width for the shamoperated control group and the AV fistula groups over the temporal course post-fistula are shown in Figure 1B. Although the LV weight was comparable to that of the sham-operated group at 5 days post-fistula, a significant decrease in cardiomyocyte length relative to the corresponding sham-operated control was distinctly evident. This decrease in cell length was a transient event with cardiomyocyte length quickly returning to essentially normal values in the subsequent period between 7 and 35 days post-fistula. The first instance that myocyte length was significantly greater than the initial baseline value was at 56 days post-fistula. Closer examination of cardiomyocyte length at 56 days post-fistula revealed a bimodal distribution, with a decompensated group in which significant in-series sarcomeric addition had occurred and another subset that remained compensated (cell lengths of $181.0 \pm 6.4$ vs. $122.4 \pm 3.2$, respectively, $P \leq$ $0.001)$. Cell length in the compensated 56 day fistula group was comparable to that in the control group. Conversely, cardiomyocyte width remained unchanged throughout the 56 days of volume overload (Figure 1B). This was also reflected by the mean cardiomyocyte diameter, which was not significantly different from the age matched sham-operated control group (22.3 \pm 1.5 vs. $21.3 \pm 2.4 \mu \mathrm{m}$ and $25.0 \pm 1.7 \mathrm{vs} .24 .0 \pm 1.8 \mu \mathrm{m}$ for the 5 day and 56 day fistula and control groups, respectively, Figure 2A-D).

\section{Cardiomyocyte Morphology}

Average sarcomere length and the number of sarcomeres present in LV cardiomyocytes from the shamoperated control and 5-day fistula groups are compared in Figure 3. It is apparent from the similar sarcomere length seen in both groups in Figure 3A that the decrease in cardiac myocyte length (Figure 1B) was not due to a change in sarcomere length. Rather, the average number 
A

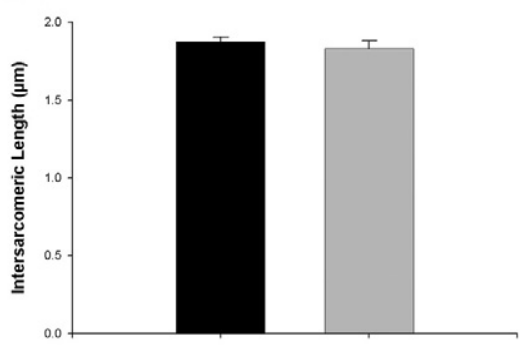

C
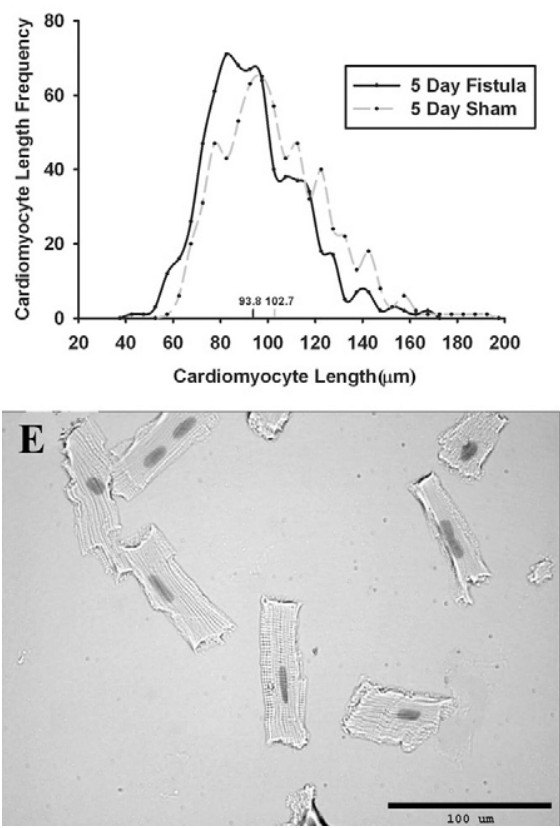

B

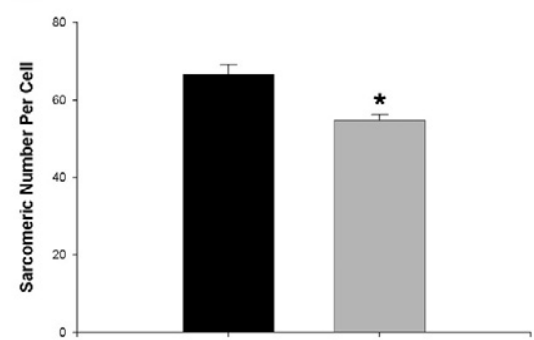

D
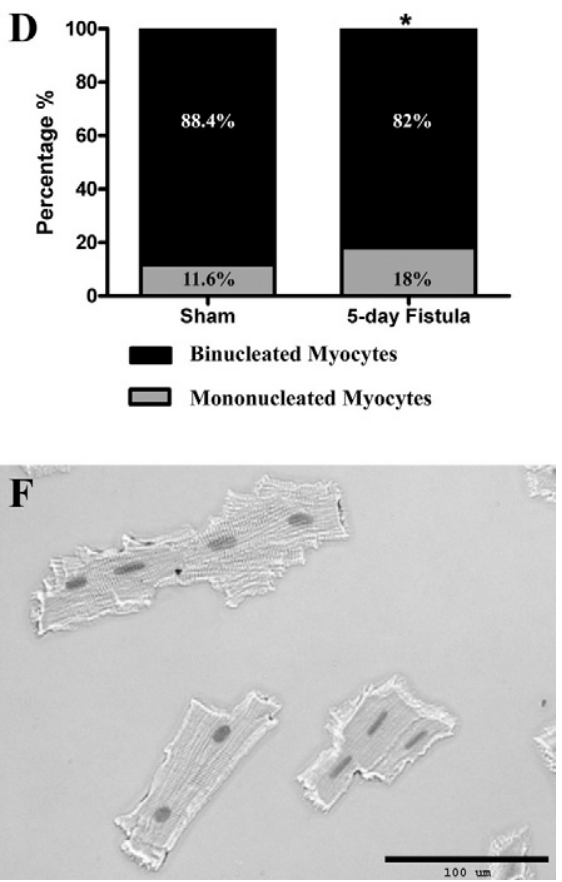

Figure 3. A comparison of average intersarcomeric length (A) and number of sarcomeres per cell (B) in left ventricular cardiomyocytes isolated from aortocaval fistula and sham-operated controls at five days post-surgery. Panel $\mathbf{C}$ contains a histogram detailing the distribution of relative length frequency obtained for left ventricular cardiomyocytes isolated from aortocaval fistula and age matched sham-operated controls at five days post-surgery. Reference lines for each curve depict median cardiomyocyte length. Panel $\mathbf{D}$ depicts the percentage of mononucleated and binucleated cardiomyocytes isolated from aortocaval fistula and age matched shamoperated controls at five days post-surgery. Concurrent with the significant decrease in cell length at five days post-fistula, there was a significant increase in the number of mononucleated cells. This is also reflected in representative photomicrographs of cardiomyocyte populations obtained following $\mathrm{KOH}$ isolation, which demonstrate a higher percentage of shorter mononucleated cardiomyocytes obtained from five day aortocaval fistula hearts (E). In contrast, the cells obtained from sham-operated control hearts consisted of predominately binucleated rod-shaped cardiomyocytes $(\mathbf{F})$. Scale bar $=100$ $\mu \mathrm{m}$. Data are mean \pm SEM. ${ }^{*} P \leq 0.01$ vs. agematched sham-operated control. of sarcomeres per cardiac myocyte in the 5-day AV fistula group was significantly decreased with respect to the sham-operated control group (Figure 3B). This decrease in cardiomyocyte length at 5 days post-fistula is further reflected in the cardiomyocyte length frequency distribution plots presented in Figure 3C. The shift of the length frequency distribution curve to the left for cardiomyocytes from hearts at 5 days post-fistula is indicative of an increased percentage of shorter cardiomyocytes. The median cardiomyocyte length at 5 days post-fistula was 93.8 $\mu \mathrm{m}$ compared with a corresponding length of $102.7 \mu \mathrm{m}$ for cells isolated from sham-operated control hearts. The cardiomyocytes from sham-operated controls were predominately binucleated ( $\sim 88 \%$; Figure $3 \mathrm{D}$ ). Concurrent with the significant decrease in cell length at 5 days postfistula, there was a corresponding significant increase in the number of mononucleated cells from 11.6 to $18 \%$ (shamoperated controls vs. 5 days post-fistula, respectively). Representative cardiomyocytes isolated from 5-day fistula and sham-operated control hearts are depicted in Figure 3, E and $F$, respectively. Evidence of microtubular reorganization associated with nuclear division was also associated with these changes in cardiomyocyte morphology at 5 days post-fistula. Figure 4A depicts a representative photomicrograph of a cardiac myocyte in interphase, in which microtubules are organized in a radially symmetrical fashion with the distal ends extending toward the cell cortex. Conversely in Figure 4B, in a cardiomyocyte at 5 days post-fistula, microtubules are concentrated to form the mitotic spindle during telophase.

\section{Hyperplasia}

The fact that myocyte size was unchanged or decreased during the first 21 days post-fistula while LV weight was increasing suggests the occurrence of cellular hyperplasia. Accordingly, additional experiments were performed as described in the Materials and Methods section to further investigate this possibility. In the first set of experiments, no evidence of BrdU labeling was found in isolated rod-shaped cardiomyocytes at 5 and 7 days postfistula. However, as depicted in Figure 5, BrdU labeling was confirmed in 0.4 to $0.8 \%$ of the non rod-shaped cardiomyocytes (equivalent to 0.1 to $0.3 \%$ of the total number of cardiomyocytes [ 6500] per slide). The second set of experiments involved the use of the chemotherapeutic agent, paclitaxel, in an attempt to prevent the occurrence of hyperplasia. As can be seen in Figure 6, A and $B$, paclitaxel prevented the decrease in cardiomyocyte length at 5 days post-fistula, as well as the relative increase in the number of mononucleated cardiomyocytes. When administered between 5 and 7 days post-fistula, paclitaxel 

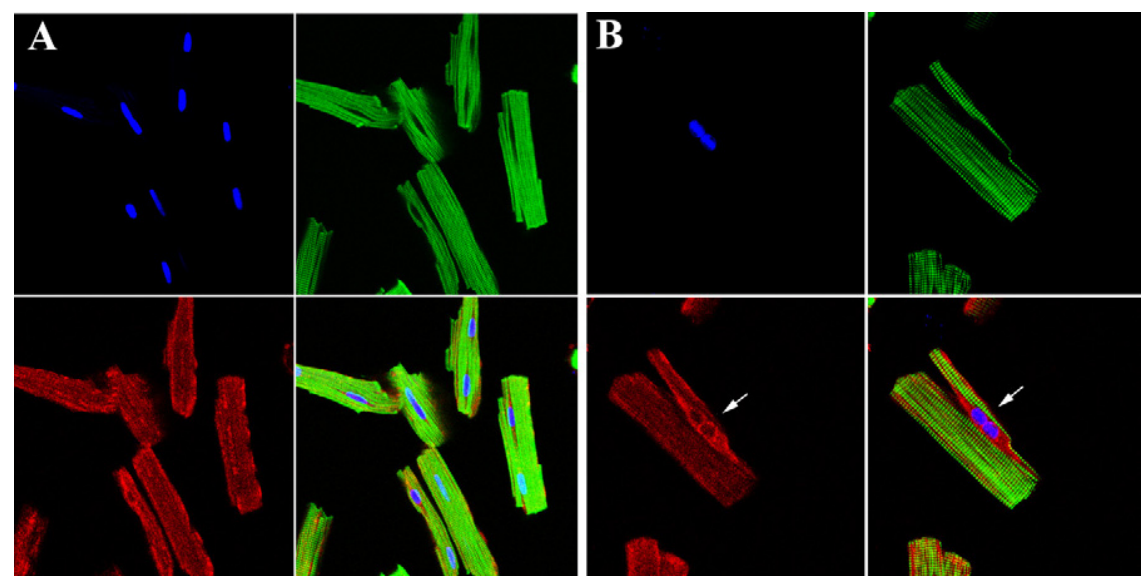

Figure 4. Representative photomicrographs demonstrating changes in microtubular organization associated with nuclear division observed in cardiomyocytes isolated from hearts at 5 days postfistula. In $\mathbf{A}$, microtubules run along the longitudinal axis of a cardiac myocyte in interphase. In B, microtubules are concentrated in the mitotic spindle during telophase. White arrow indicates mitotic spindle. Cells are triple labeled with antibodies directed against tubulin (red), F-actin (green), and the nucleus (blue). did not prevent the return to a normal percentage of monoand bi-nucleated cardiomyocytes. However, paclitaxel did appear to interfere with completion of cardiomyocyte mitosis, leading to chromosomal mis-segregation and multinucleation as depicted in Figure 6, C and D. Inhibition of cytokinesis produced a marked increase in quadrinucleated cardiomyocytes (ie, $<0.01 \%$ in untreated versus $0.20 \%$ in treated hearts, Figure $6 D$ ), reflecting adult binucleated cells reentering cell cycle.

\section{Extracellular Matrix Degradation}

Consistent with previously published observations, scanning electron micrographs from sham-operated control hearts illustrate the extensively branched arborization of collagen fibrils surrounding the cardiomyocytes, as well as collagen struts interconnecting adjacent cardiomyocytes (Figure 7, A and C). Conversely, in myocardial sections taken at 5 days post-fistula (Figure 7, B and D), the collagen latticework was disorganized and extensively degraded, with the collagen struts being for the most part absent.

\section{Discussion}

Numerous studies have documented gender differences in the pattern of global ventricular remodeling in hearts

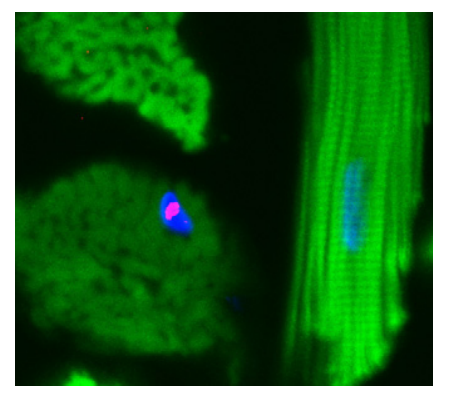

Figure 5. Representative photomicrograph depicting BrdU incorporation into the newly synthesized DNA of replicating cells. No evidence of BrdU labeling was found in rod-shaped cardiomyocytes isolated post-fistula. However, cardiomyocytes that had recently undergone cytokinesis would potentially be prone to instability due to the cytoskeletal reorganization. Cells are triple labeled with antibodies directed against BrdU (red), F-actin (green), and the nucleus (blue). subjected to a sustained increase in myocardial stress and/or injury. ${ }^{14-17}$ Historically, ventricular remodeling has been described as either concentric or eccentric hypertrophy, with pressure and volume overload inducing cardiomyocytes to remodel by either parallel or in-series addition of sarcomeres, resulting in their thickening and lengthening, respectively. ${ }^{2}$ Recent findings from our laboratory have shown myocardial remodeling in male rats is characterized by development of eccentric hypertrophy and heart failure. ${ }^{5,6}$ In contrast, female rats respond to a sustained ventricular volume overload with a concentric pattern of hypertrophy, which allows them to remain compensated. ${ }^{7-9,18}$ Gender differences in myocyte volume following a myocardial infarction, such that average myocyte volume was $36 \%$ greater in men than in women, together with a $14 \%$ increase in resting cell length, have been reported to parallel marked gender differences in LV mass. ${ }^{14}$ Consideration of these findings suggests the possibility that a difference in the response of cardiac myocytes to ventricular volume overload conditions may contribute to these disparate outcomes. Thus, this study was undertaken to determine whether cardiomyocyte remodeling in response to a sustained ventricular volume overload in males differed from that previously reported for female rats. ${ }^{3,4}$

The key findings of this study confirm a gender associated difference in the remodeling of cardiomyocytes that is due at least in part to the occurrence of cardiomyocyte hyperplasia in male hearts during the first 5 days of volume overload. Specifically, we found cardiomyocytes in male hearts underwent a transient but significant decrease in cell length at 5 days post-fistula. A concurrent increase in the ratio of mononucleated to binucleated cardiomyocytes and a reduction in the number of sarcomeres per cardiomyocyte relative to sham-operated control hearts were associated with the decrease in cardiomyocyte length observed at 5 days post-fistula. However, the shift in the ratio of mono- to bi-nucleated cells, as well as the decrease in length was transient, with the nuclear ratio and cardiomyocyte length having returned to normal by 7 days post-fistula. Cardiomyocyte width and crosssectional area did not vary significantly throughout the study period. Although the ventricle underwent continued remodeling (ie, as reflected by increased LV mass), car- 
A
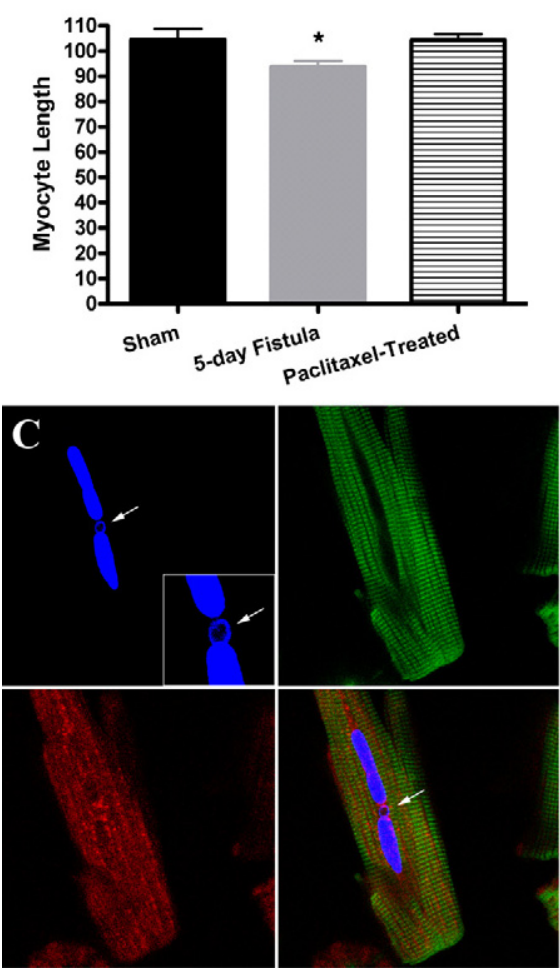

B
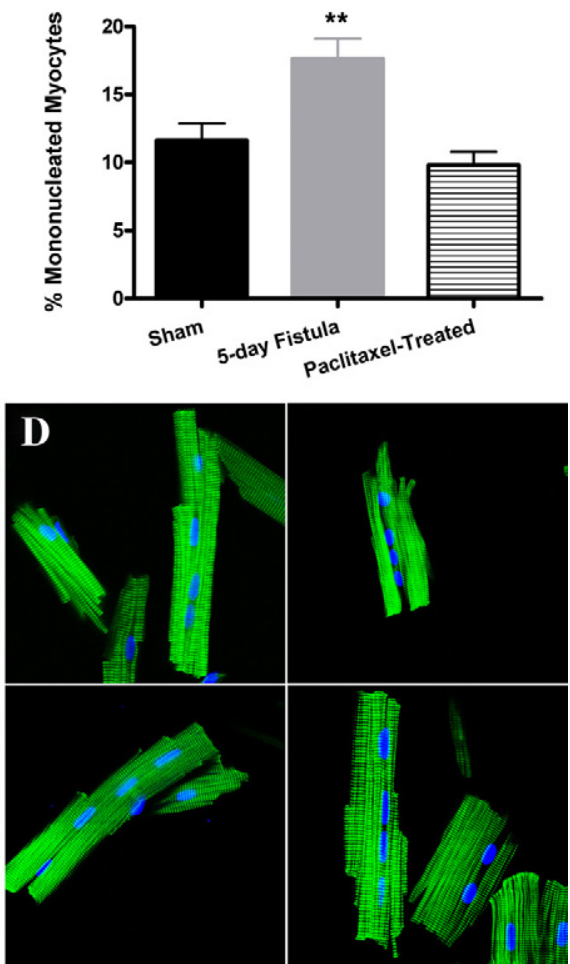

Figure 6. The effects of microtubule stabilization with paclitaxel (taxol) can be seen in these graphs depicting the changes in average cardiomyocyte length (A) and the percentage of mononucleated cardiomyocytes (B) in isolated cells obtained from aortocaval fistula and age matched sham-operated control rats at five days post-surgery. Treatment with paclitaxel prevented both the decrease in cardiomyocyte length and the relative increase in the number of mononucleated cardiomyocytes at five days post-fistula Paclitaxel appeared to interfere with completion of cardiomyocyte mitosis and cytokinesis. An example of chromosomal mis-segregation is depicted in $\mathbf{C}$ (indicated by white arrow), wherein a defect in chromosome segregation produced by one or more lagging chromosomes separated from the bulk of DNA clusters leads to micronuclear formation. Panel $\mathbf{D}$ depicts several examples of quadrinucleated cardiomyocytes induced when cytokinesis was inhibited by administration of paclitaxel between five and seven days post-fistula. Cells are labeled with antibodies directed against tubulin (red), F-actin (green), and/or the nucleus (blue). Data are mean \pm SEM. ${ }^{*} P \leq 0.05$ vs. age-matched shamoperated control; Two total ${ }^{* *} P \leq 0.01$ vs. agematched sham-operated control. diomyocyte length remained relatively constant during the compensated phase of myocardial remodeling, not exceeding normal values until sometime after 35 days post-fistula. In contrast, the temporal profile for LV mass was distinctly different, with substantial progressive increases occurring over the same 35-day period (Figure 1). These observations in male hearts are in stark contrast to the remodeling reported in female hearts, where continuous proportional increases in cardiomyocyte length
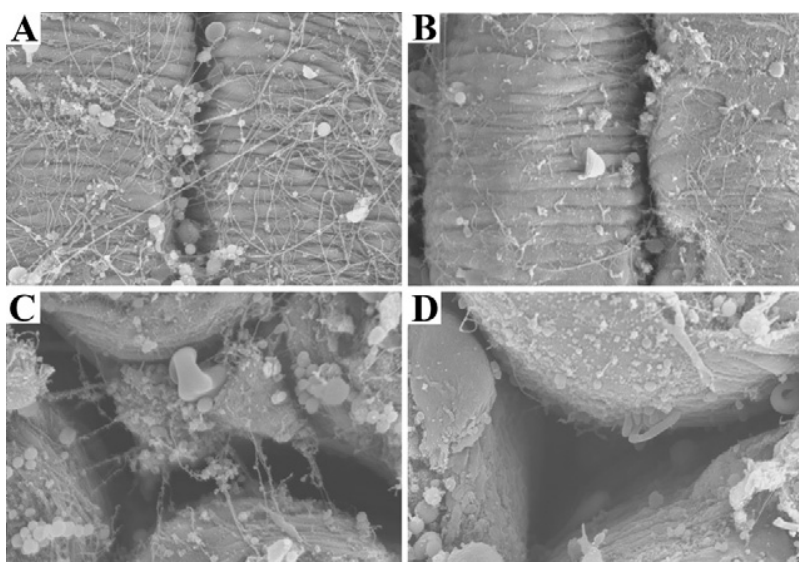

Figure 7. Representative scanning electron micrographs depicting the typical organization of myocardial extracellular matrix present in sham-operated controls (A and $\mathbf{C}$ ), illustrate the extensively branched arborization of collagen fibrils surrounding the cardiomyocytes, as well as collagen struts interconnecting adjacent cardiomyocytes. Contrast this with the micrographs taken of myocardial sections at five days post-fistula ( $\mathbf{B}$ and $\mathbf{D})$, in which the collagen latticework was extensively degraded, with the collagen struts interconnecting cardiomyocytes being for the most part absent and width occurred over the entire temporal course postfistula from 5 days through 6 months. ${ }^{3,4,19,20}$

This significant trend toward a greater number of smaller, mononucleated cardiomyocytes occurring without a concomitant reduction in LV weight can only be interpreted as hyperplasia resulting from 1) division of differentiated adult cells or 2) myocyte proliferation and/or the generation of new cardiomyocytes from stem cells. We next performed experiments using BrdU and paclitaxel to determine the source of the increased number of shorter, mononucleated cardiomyocytes in males. $\mathrm{BrdU}$ is permanently incorporated into the newly synthesized DNA of replicating cells, substituting for thymidine during DNA replication in the $S$ phase of the cell cycle. Accordingly, BrdU labeling is indicative of cells that were actively replicating their DNA during the period of exposure. In contrast, paclitaxel binds to $\beta$ tubulin and interferes with the normal function of microtubule depolymerization. ${ }^{12}$ This adversely affects cell division because the dynamic instability of microtubules is necessary for accurate chromosome segregation and subsequent cytokinesis. ${ }^{21,22}$ Accordingly, paclitaxel-induced stabilization of microtubules results in chromosome mis-segregation, formation of micronuclei, and multinucleated cells as a result of irregular mitosis and cytokinesis. Although rodshaped cardiomyocytes isolated from hearts at 5 days post-fistula were not positive for BrdU labeling, a small percentage of rounded cardiomyocytes did incorporate BrdU labeling in the nucleus, indicating that the extent of DNA replication and nuclear division that did occur was insufficient to produce the significant increase in the number of shorter mononucleated cells. However, the 
data suggests that cytokinesis of adult binucleated cells could be the mechanism, since inhibition of cytokinesis with paclitaxel administered from 0 to 5 days post-fistula prevented the increase in the number of shorter mononucleated cardiomyocytes. The subsequent normalization of the ratio of mononucleated to binucleated cardiomyocytes suggests the possibility that karyokinesis was taking place after the initial cellular division. That this was indeed occurring is reflected by the substantial increase in quadrinucleated cells seen with paclitaxel between 5 and 7 days post-fistula (Figure 6). Taken together, these novel observations strongly implicate cytokinesis of binucleated adult cardiomyocytes, occurring without initial nuclear division, as the source of cardiomyocyte hyperplasia.

Cardiomyocyte proliferation is another possibility that must be considered. However, only 0.1 to $0.3 \%$ of the cardiomyocytes on a given slide were found to be BrdU positive, and these were not rod shaped cardiomyocytes. We surmise that the cytoskeleton in these newly generated cells was less stable, making them prone to damage during the enzymatic isolation, consequently resulting in a rounded appearance, rather than a cardiomyocyte's typical rod shaped configuration. Although the percentage of rounded BrdU positive cardiomyocytes obtained in this study was somewhat higher than that reported in postmortem examination of human hearts following a myocardial infarction $(0.03 \%$ in remote myocardium and $0.08 \%$ in infarct border zone versus $0.001 \%$ in normal hearts), ${ }^{23}$ this level of proliferation still could not account for the increase in mononucleated cardiomyocytes from 11.6 to $18 \%$ observed in the male hearts post-fistula. Likewise, while stem cells cannot be discounted as a source contributing to these new mononucleated cells, it is doubtful based on the representative literature that they could generate a $6 \%$ increase in such a short time. ${ }^{24-29}$ For example, Beltrami et $\mathrm{al}^{25}$ reported a ratio of one cardiac stem cell for every 10,000 cardiomyocytes or $0.01 \%$. Another recent study established the capacity to generate new cardiomyocytes in the adult human heart was limited to a rate $\leq 1 \%$ of the myocardium per year. ${ }^{30}$ Thus, while cardiomyocytes originating from proliferation of stem cells may have contributed to the increase in the percentage of mononucleated cardiomyocytes, we would again conclude that most of the increase resulted from cytokinesis of binucleated cardiomyocytes without nuclear division.

The concept that cardiomyocytes were terminally differentiated and could not reenter cell cycle became dogma in the early Twentieth Century. ${ }^{31}$ However, in 1960, Linzbach ${ }^{32}$ reintroduced the concept that cellular hyperplasia may contribute to the pathological increase in myocardial mass. Several intervening studies have contributed additional evidence supporting the supposition that adult cardiomyocytes have the potential to reenter cell cycle and proliferate. ${ }^{23,26,27,33-44}$ The findings herein indicate that hyperplasia in male hearts, rather than hypertrophy, is the predominant mechanism contributing to the significant increase in LV mass during the compensated LV hypertrophy characteristic of this model of heart failure. ${ }^{5,6}$ This would also be consistent with the study by Grajek et al, ${ }^{38}$ which found evidence of hyper- plasia in a postmortem morphometric study of human hearts reflected by a significant increase in the number of cardiomyocytes in hearts with LV weight exceeding $250 \mathrm{~g}$. However, we have previously established that there appears to be an upper maximal limit to the hypertrophic response post-fistula. ${ }^{6,45}$ This appears to coincide with the onset of cardiomyocyte elongation, reflecting a transition to in-series sarcomeric addition seen at 56 days post-fistula. Although the trigger for the transition in the pattern of cardiomyocyte remodeling from hyperplasia to in-series sarcomeric addition is not clear, it appears to be associated with the subsequent development of ventricular dilatation and heart failure, which consistently develops in males as early as 56 days post-fistula. ${ }^{5,6,45,46}$ Furthermore, this observation is consistent with the pathological remodeling described by Linzbach and others ${ }^{32,47-49}$ when the heart exceeds a "critical heart weight" that underlies the development of ventricular dilatation. This significant increase in cardiomyocyte length, together with minimal changes in width, is characteristic of the cardiomyocyte remodeling commonly associated with failing hearts. ${ }^{50-52}$

\section{Perspective on Mechanisms}

As a result of our careful characterization of the aortocaval fistula model, three distinct phases of myocardial remodeling have been identified. ${ }^{45}$ The initial phase, which encompasses the first week of chronic volume overload, is characterized by a marked increase in matrix metalloproteinase (MMP) activity, producing extensive degradation of the fibrillar collagen matrix in the heart ( $48 \%$ and $61 \%$ at 3 and 5 days post-fistula, respectively), thus allowing for ventricular dilatation. ${ }^{53,54}$ The extensive nature of the extracellular matrix (ECM) degradation is depicted in Figure 7. Beyond approximately 2 weeks post-fistula, the heart transitions to a chronic compensatory phase during which MMP activity and collagen concentration return to normal, together with a progressive increase in LV mass (Figure 1). However, at or about 8 weeks post-fistula, the transition to the decompensated phase develops. ${ }^{6,54}$ The decompensated phase is characterized by marked ventricular dilatation associated with significant increases in MMP activity and ventricular compliance, as well as a significant reduction in systolic function. ${ }^{6}$ The duration of the remodeling process is variable, with some rats entering the decompensated phase as early as 5 weeks post-fistula, while others remain compensated for longer periods, with morbidity/mortality due to heart failure reaching $80 \%$ at 20 weeks of chronic volume overload. This is reflected by the data herein, with approximately half of the rats in the 56 day group being decompensated and having significant increases in cardiomyocyte length, whereas cardiomyocyte length in the remaining compensated hearts was comparable to that in the sham-operated controls.

The significant differences between our results obtained in males compared with those reported by ourselves and others in females clearly indicate a gender difference in the response of the heart to a sustained elevation in myocardial stress. ${ }^{3-9,18-20,55}$ This difference 
may be partially related to the fact that estrogen has been shown to phenotypically modify the cardiac mast cell, whereby it lacked the secretory product(s) that are capable of activating MMPs. This was shown by Chancey et $\mathrm{al},{ }^{56}$ where chemical induction of myocardial mast cell degranulation resulted in a rapid increase in MMP activity (within 30 minutes) and a corresponding 50\% reduction in collagen concentration in hearts from male and ovariectomized female rats. In contrast, even though $98 \%$ of the mast cells were found to be degranulated in all groups, MMP activity and collagen concentration were not affected in hearts from intact and estrogen-treated ovariectomized females. As a result of the alterations to the ECM in hearts from male and ovariectomized female rats, there was a significant rightward shift in the LV end diastolic pressure-volume relation, indicating structural ventricular dilatation.

The possibility that estrogen mediates the cardioprotective effects conferred by gender prompted us to evaluate the structural and functional effects of estrogen in male rats subjected to chronic volume overload. ${ }^{57} \mathrm{Al}-$ though both fistula groups developed significant ventricular hypertrophy after 8 weeks of volume overload, estrogen attenuated LV dilatation and maintained function in treated rats. Estrogen treatment was also associated with prevention of MMP activation and the breakdown of ventricular collagen in the early stage of remodeling. These data demonstrate that estrogen attenuates ventricular remodeling and disease progression in male rats subjected to chronic volume overload. These observations, coupled with the fact that cardiac mast cells have been shown to be responsible for the increase in myocardial MMP activity and collagen degradation in male hearts during the initial phase following the creation of an AV fistula, ${ }^{45,53,54}$ indicate that the gender difference in myocyte remodeling at 5 days and beyond may be the result of myocardial ECM degradation that occurs in males.

Integrins are transmembrane receptors that serve as the primary linkage between their ECM ligands and the cytoskeleton. Integrins perceive changes in the extracellular environment, and by transmitting chemical and mechanical information into the cell initiate intracellular signaling cascades and gene transcription. ${ }^{58,59}$ Thus cardiomyocyte perception of the ECM degradation could be responsible for the cytokinesis of binucleated cardiomyocytes without nuclear division. For example, small GTPases, such as Rho-kinase/ROCK molecules, which are downstream of integrin mediated signal transduction, ${ }^{58}$ have been recognized in regulation of cleavage furrow formation necessary for completion of cytokinesis. ${ }^{60,61}$ RhoA GTPase is another key molecule regulating contractile ring assembly in cytokinesis. ${ }^{62}$ Given the extensive ECM degradation taking place in the initial 5 days post-fistula, it is entirely plausible that altered integrin mediated signal transduction could initiate cardiomyocyte cytokinesis.

In summary, the findings herein indicate that hyperplasia resulting from cytokinesis of differentiated cardiomyocytes is a key mechanism, independent of hypertrophy, contributing to the significant increase in LV mass in male hearts subjected to chronic volume overload. Furthermore, these observations confirm that marked gender differences at the level of the cardiomyocyte may transform the myocardial remodeling process, ultimately causing the transition to heart failure. This gender difference in the pattern of myocardial remodeling may be related to the degradation of the ECM that is known to occur in male hearts during the initial remodeling post-fistula. Although the resulting increase in LV mass due to hyperplasia in males initially contributes to maintaining compensated ventricular function, the eventual development of heart failure is preceded by the transition to in-series sarcomeric addition. Future studies should attempt to clarify the mechanism by which female hormone(s), such as estrogen, contribute to gender differences in cardiomyocyte cellular hypertrophy versus hyperplasia. It is also equally important to identify the specific factor(s) mediating the transition to in-series sarcomeric addition responsible for the development of ventricular dilatation and heart failure.

\section{Acknowledgment}

We would like to thank Mr. Jeffrey Davis for excellent technical assistance.

\section{References}

1. Grossman W, Jones D, McLaurin LP: Wall stress and patterns of hypertrophy in the human left ventricle. J Clin Invest 1975, 56:56-64

2. Opie LH, Commerford PJ, Gersh BJ, Pfeffer MA: Controversies in ventricular remodelling. The Lancet 367:356-367

3. Liu Z, Hilbelink DR, Crockett WB, Gerdes AM: Regional changes in hemodynamics and cardiac myocyte size in rats with aortocaval fistulas. 1. Developing and established hypertrophy. Circ Res 1991, 69:52-58

4. Liu Z, Hilbelink DR, Gerdes AM: Regional changes in hemodynamics and cardiac myocyte size in rats with aortocaval fistulas. 2. Long-term effects. Circ Res 1991, 69:59-65

5. Brower GL, Henegar JR, Janicki JS: Temporal evaluation of left ventricular remodeling and function in rats with chronic volume overload Am J Physiol Heart Circ Physiol 1996, 271:H2071-H2078

6. Brower GL, Janicki JS: Contribution of ventricular remodeling to pathogenesis of heart failure in rats. Am J Physiol Heart Circ Physiol 2001, 280:H674-H683

7. Brower GL, Gardner JD, Janicki JS: Protection from adverse cardiac remodeling induced by chronic volume overload is abolished by ovariectomy. Mol Cell Biochem 2003, 251:89-95

8. Gardner JD, Brower GL, Janicki JS: Gender differences in cardiac remodeling secondary to chronic volume overload. J Card Fail 2002 8:101-107

9. Gardner JD, Brower GL, Voloshenyuk TG, Janicki JS: Cardioprotection in female rats subjected to chronic volume overload: synergistic interaction of estrogen and phytoestrogens. Am J Physiol Heart Circ Physiol 2008, 294:H198-H204

10. Gerdes AM, Onodera T, Tamura T, Said S, Bohlmeyer TJ, Abraham WT, Bristow MR: New method to evaluate myocyte remodeling from formalinfixed biopsy and autopsy material. J Card Fail 1998, 4:343-348

11. Tieman SB. Gallocyanin-chrome alum counterstaining of GolgiKopsch impregnations. Stain Technol 1983, 58:171-175

12. Schiff PB, Fant J, Horwitz SB: Promotion of microtubule assembly in vitro by taxol. Nature 1979, 277:665-667

13. Murphy JA: Non-coating techniques to render biological specimens conductive/1980 update. Scanning Electron Microscopy. Part I. Edited by O. Johari. Chicago IL, 1980, pp 209-220

14. Crabbe DL, Dipla K, Ambati S, Zafeiridis A, Gaughan JP, Houser SR, Margulies KB: Gender differences in post-infarction hypertrophy in end-stage failing hearts. J Am Coll Cardiol 2003, 41:300-306

15. Vasan RS, Evans JC, Benjamin EJ, Levy D, Larson MG, Sundstrom J, Murabito JM, Sam F, Colucci WS, Wilson PWF: Relations of serum 
aldosterone to cardiac structure: gender-related differences in the Framingham heart study. Hypertension 2004, 43:957-962

16. Luchner A, Brockel U, Muscholl M, Hense HW, Doring A, Riegger GAJ, Schunkert H: Gender-specific differences of cardiac remodeling in subjects with left ventricular dysfunction: a population-based study. Cardiovasc Res 2002, 53:720-727

17. Villar AV, Llano M, Cobo M, Expósito V, Merino R, Martín-Durán R, Hurlé MA, Nistal JF: Gender differences of echocardiographic and gene expression patterns in human pressure overload left ventricular hypertrophy. J Mol Cell Cardiol 2009, 46:526-535

18. Gardner JD, Brower GL, Janicki JS: Effects of dietary phytoestrogens on cardiac remodeling secondary to chronic volume overload in female rats. J Appl Physiol 2005, 99:1378-1383

19. Hatt PY, Rakusan K, Gastineau P, Laplace M: Morphometry and ultrastructure of heart hypertrophy induced by chronic volume overload (aorto-caval fistula in the rat). J Mol Cell Cardiol 1979, 11:988-998

20. Hatt PY, Rakusan K, Gastineau P, Laplace M, Cluzeaud F: Aortocaval fistula in the rat. An experimental model of heart volume overloading. Basic Res Cardiol 1980, 75:105-108

21. Strickland LI, Donnelly EJ, Burgess DR: Induction of cytokinesis is independent of precisely regulated microtubule dynamics. Mol Biol Cell 2005, 16:4485-4494

22. Zhou J, Giannakakou P: Targeting microtubules for cancer chemotherapy. Curr Med Chem Anticancer Agents 2005, 5:65-71

23. Beltrami AP, Urbanek K, Kajstura J, Yan SM, Finato N, Bussani R, Nadal-Ginard B, Silvestri F, Leri A, Beltrami CA, Anversa P: Evidence that human cardiac myocytes divide after myocardial infarction. The N Engl J Med 2001, 344:1750-1757

24. Anversa $P$, Nadal-Ginard B: Myocyte renewal and ventricular remodelling. Nature 2002, 415:240-243

25. Beltrami AP, Barlucchi L, Torella D, Baker M, Limana F, Chimenti S, Kasahara H, Rota M, Musso E, Urbanek K, Leri A, Kajstura J, NadalGinard B, Anversa P: Adult cardiac stem cells are multipotent and support myocardial regeneration. Cell 2003, 114:763-776

26. Engel FB: Cardiomyocyte proliferation: a platform for mammalian cardiac repair. Cell Cycle 2005, 4:1360-1363

27. Kajstura J, Pertoldi B, Leri A, Beltrami CA, Deptala A, Darzynkiewicz $Z$, Anversa P: Telomere shortening Is an in vivo marker of myocyte replication and aging. Am J Pathol 2000, 156:813-819

28. Kajstura J, Urbanek K, Rota M, Bearzi C, Hosoda T, Bolli R, Anversa P, Leri A: Cardiac stem cells and myocardial disease. J Mol Cell Cardiol 2008, 45:505-513

29. Leri A, Kajstura J, Anversa P: Cardiac stem cells and mechanisms of myocardial regeneration. Physiol Rev 2005, 85:1373-1416

30. Bergmann O, Bhardwaj RD, Bernard S, Zdunek S, Barnabe-Heider F, Walsh S, Zupicich J, Alkass K, Buchholz BA, Druid H, Jovinge S Frisen J: Evidence for cardiomyocyte renewal in humans. Science 2009, 324:98-102

31. Anversa P, Leri A, Kajstura J: Cardiac regeneration. J Am Coll Cardiol 2006, 47:1769-1776

32. Linzbach AJ: Heart failure from the point of view of quantitative anatomy. Am J Cardiol 1960, 5:370-382

33. Anversa P, Kajstura J: Ventricular myocytes are not terminally differentiated in the adult mammalian heart. Circ Res 1998, 83:1-14

34. Beltrami CA, Di Loreto C, Finato N, Rocco M, Artico D, Cigola E, Gambert SR, Olivetti G, Kajstura J, Anversa P: Proliferating cell nuclear antigen (PCNA). DNA synthesis and mitosis in myocytes following cardiac transplantation in man. J Mol Cell Cardiol 1997, 29:2789-2802

35. Bicknell KA, Coxon CH, Brooks G: Can the cardiomyocyte cell cycle be reprogrammed? J Mol Cell Cardiol 2007, 42:706-721

36. Engel FB, Schebesta M, Duong MT, Lu G, Ren S, Madwed JB, Jiang H, Wang $Y$, Keating MT: p38 MAP kinase inhibition enables proliferation of adult mammalian cardiomyocytes. Genes Dev 2005, 19:1175-1187

37. Engel FB, Hauck L, Cardoso MC, Leonhardt H, Dietz R, von Harsdorf R: A mammalian myocardial cell-free system to study cell cycle reentry in terminally differentiated cardiomyocytes. Circ Res 1999 85:294-301

38. Grajek S, Lesiak M, Pyda M, Zajac M, Paradowski S, Kaczmarek E Hypertrophy or hyperplasia in cardiac muscle. Post-mortem human morphometric study. Eur Heart J 1993, 14:40-47

39. Kajstura J, Leri A, Finato N, Di LC, Beltrami CA, Anversa P: Myocyte proliferation in end-stage cardiac failure in humans. Proc Natl Acad Sci USA 1998, 95:8801-8805
40. Nadal-Ginard B, Kajstura J, Leri A, Anversa P: Myocyte death, growth, and regeneration in cardiac hypertrophy and failure. Circ Res 2003, 92:139-150

41. Olivetti G, Quaini F, Lagrasta C, Ricci R, Tiberti G, Capasso JM, Anversa P: Myocyte cellular hypertrophy and hyperplasia contribute to ventricular wall remodeling in anemia-induced cardiac hypertrophy in rats. Am J Pathol 1992, 141:227-239

42. Olivetti G, Melissari M, Balbi T, Quaini F, Sonnenblick EH, Anversa P. Myocyte nuclear and possible cellular hyperplasia contribute to ventricular remodeling in the hypertrophic senescent heart in humans. J Am Coll Cardiol 1994, 24:140-149

43. van Amerongen MJ, Engel FB: Features of cardiomyocyte proliferation and its potential for cardiac regeneration. J Cell Mol Med 2008, 12:2233-2244

44. Zaglia T, Dedja A, Candiotto C, Cozzi E, Schiaffino S, Ausoni S: Cardiac interstitial cells express GATA4 and control dedifferentiation and cell cycle re-entry of adult cardiomyocytes. J Mol Cell Cardiol 2009, 46:653-662

45. Janicki JS, Brower GL, Gardner JD, Chancey AL, Stewart Jr JA: The dynamic interaction between matrix metalloproteinase activity and adverse myocardial remodeling. Heart Fail Rev 2004, 9:33-42

46. Brower GL, Janicki JS: Pharmacologic inhibition of mast cell degranulation prevents left ventricular remodeling induced by chronic volume overload in rats. J Card Fail 2005, 11:548-556

47. Buja LM, Vela D: Cardiomyocyte death and renewal in the normal and diseased heart. Cardiovasc Pathol 2008, 17:349-374

48. Francis GS: Heart failure. J Am Coll Cardiol 1999, 33:291-294

49. Linzbach AJ: Hypertrophy, hyperplasia and structural dilatation of the human heart. Advanced Cardiol 1976, 18:1-14

50. Beltrami CA, Finato N, Rocco M, Feruglio GA, Puricelli C, Cigola E, Quaini F, Sonnenblick EH, Olivetti G, Anversa P: Structural basis of end-stage failure in ischemic cardiomyopathy in humans. Circulation 1994, 89:151-163

51. Onodera T, Tamura T, Said S, McCune SA, Gerdes AM: Maladaptive remodeling of cardiac myocyte shape begins long before failure in hypertension. Hypertension 1998, 32:753-757

52. Zafeiridis A, Jeevanandam V, Houser SR, Margulies KB: Regression of cellular hypertrophy after left ventricular assist device support. Circulation 1998, 98:656-662

53. Brower GL, Chancey AL, Thanigaraj S, Matsubara BB, Janicki JS Cause and effect relationship between myocardial mast cell number and matrix metalloproteinase activity. Am J Physiol Heart Circ Physiol 2002, 283:H518-H525

54. Janicki JS, Brower GL, Gardner JD, Forman MF, Stewart JA, Jr. Murray DB, Chancey AL: Cardiac mast cell regulation of matrix metalloproteinase-related ventricular remodeling in chronic pressure or volume overload. Cardiovasc Res 2006, 69:657-665

55. Chancey AL, Brower GL, Janicki JS: Cardiac mast cell-mediated activation of gelatinase and alteration of ventricular diastolic function. Am J Physiol Heart Circ Physiol 2002, 282:H2152-H2158

56. Chancey AL, Gardner JD, Murray DB, Brower GL, Janicki JS: Modulation of cardiac mast mediated extracellular matrix degradation by estrogen. Am J Physiol Heart Circ Physiol 2005, 289:H316-H321

57. Gardner JD, Murray DB, Voloshenyuk TG, Brower GL, Bradley JM, Janicki JS: Estrogen attenuates chronic volume overload induced structural and functional remodeling in male rat hearts. Am J Physiol Heart Circ Physiol 2010, 298:H497-H504

58. Ross RS, Borg TK: Integrins and the myocardium. Circ Res 2001, 88:1112-1119

59. Sussman MA, McCulloch A, Borg TK: Dance band on the Titanic: biomechanical signaling in cardiac hypertrophy. Circ Res 2002, 91: $888-898$

60. Lamarche N, Tapon N, Stowers L, Burbelo PD, Aspenström P, Bridges T, Chant J, Hall A: Rac and Cdc42 induce actin polymerization and G1 cell cycle progression independently of p65PAK and the JNK/SAPK MAP kinase cascade. Cell 1996, 87:519-529

61. Yokoyama T, Goto H, Izawa I, Mizutani H, Inagaki M: Aurora-B and Rho-kinase/ROCK, the two cleavage furrow kinases, independently regulate the progression of cytokinesis: possible existence of a nove cleavage furrow kinase phosphorylates ezrin/radixin/moesin (ERM). Genes Cells 2005, 10:127-137

62. Glotzer M: The molecular requirements for cytokinesis. Science 2005 307:1735-1739 